SUBMitTed TO The Astrophysical Journal

Preprint typeset using LTEX style emulateapj v. 11/10/09

\title{
A SHOCK FRONT IN THE MERGING GALAXY CLUSTER ABELL 754: X-RAY AND RADIO OBSERVATIONS
}

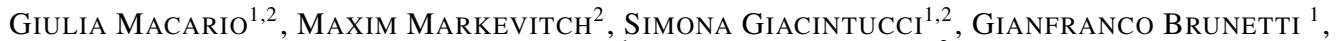 \\ TIZIANA VENTURI ${ }^{1}$, STEPHEN S. MURRAY ${ }^{2}$ \\ submitted to The Astrophysical Journal
}

\begin{abstract}
We present new Chandra X-ray and Giant Meterwave Radio Telescope (GMRT) radio observations of the nearby merging galaxy cluster Abell 754. Our X-ray data confirm the presence of a shock front by obtaining the first direct measurement of a gas temperature jump across the X-ray brightness edge previously seen in the imaging data. A 754 is only the fourth galaxy cluster with confirmed merger shock fronts, and it has the weakest shock of those, with a Mach number $\mathrm{M}=1.57_{-0.12}^{+0.16}$. In our new $G M R T$ observation at $330 \mathrm{MHz}$, we find that the previously-known centrally located radio halo extends eastward to the position of the shock. The X-ray shock front also coincides with the position of a radio relic previously observed at $74 \mathrm{MHz}$. The radio spectrum of the post-shock region, using our radio data and the earlier results at $74 \mathrm{MHz}$ and $1.4 \mathrm{GHz}$, is very steep. We argue that acceleration of electrons at the shock front directly from thermal to ultrarelativistic energies is problematic due to energy arguments, while reacceleration of preexisting relativistic electrons is more plausible.

Subject headings: galaxies: clusters: general — galaxies: clusters: individual (A754) — intergalactic medium - radio continuum: galaxies - $\mathrm{X}$-rays: galaxies: clusters
\end{abstract}

\section{INTRODUCTION}

Galaxy clusters form via mergers of smaller substructures. During such mergers, most of the kinetic energy of the gas belonging to the colliding subclusters is dissipated by shocks and turbulence into thermal energy of the intracluster medium (ICM) of the resulting system. Shocks and turbulence are also expected to amplify the cluster magnetic fields and accelerate cosmic ray particles from the thermal ICM, or reaccelerate preexisting relativistic particles. These non-thermal components manifest themselves as diffuse synchrotron radio sources, known as radio halos and relics (e.g., Ferrari et al. 2008; Cassano 2009 for recent reviews), and inverse Compton X-ray emission at high energies (e.g., Fusco-Femiano et al. 2004; Rephaeli \& Gruber 2002; see, however, Wik et al. 2009).

Shock fronts represent a unique observational tool to study the physical processes in the ICM. They create sharp discontinuities in the cluster X-ray surface brightness images and allow to measure the gas velocities in the sky plane using X-ray imaging spectroscopy (e.g., Markevitch, Sarazin, \& Vikhlinin 1999). Shock-heated regions are routinely observed in merging clusters. However, known shock fronts are still rare, because one has to catch the front when it has not yet moved to the outer, low surface brightness regions of the cluster, where the background X-ray emission dominates. Moreover, the merger has to be occurring nearly in the plane of the sky, otherwise projection effects could hide the gas density and temperture jumps.

Until now, reliable detections of merger shock fronts have been reported in only three galaxy clusters. One is the Bullet cluster (1E 0657-56, Markevitch et al. 2002), another is Abell 520 (Markevitch et al. 2005), and the two fronts have been recently discovered in Abell 2146 (Russell et al. 2010). Only in these clusters both the sharp gas density edges and

\footnotetext{
${ }^{1}$ INAF/Istituto di Radioastronomia, via Gobetti 101, I-40129, Bologna, Italy; g.macario@ira.inaf.it

${ }^{2}$ Harvard-Smithsonian Center for Astrophysics, 60 Garden Street, Cambridge, MA 02138, USA
}

the unambiguous temperature jumps were found, allowing the identification of the brightness feature as a shock and determination of the gas velocity. In this paper, we report on the new shock front in the merging cluster Abell 754. We present the analysis of Chandra observations and study the connection between the shock and the diffuse cluster radio emission, through Giant Metrewave Radio Telescope (GMRT) observations at $330 \mathrm{MHz}$ and Very Large Array (VLA) archival data at $1.4 \mathrm{GHz}$.

We assume a flat cosmology with $\mathrm{H}_{0}=70 \mathrm{~km} \mathrm{~s}^{-1} \mathrm{Mpc}^{-1}$ and $\Omega_{0}=0.3$, in which $1^{\prime \prime}$ is $1.054 \mathrm{kpc}$ at the redshift of A754. We adopt the convention $S \propto \nu^{-\alpha}$ for the synchrotron spectrum. Uncertainties are $68 \%$, unless stated otherwise.

\section{THE MERGING CLUSTER A754}

A754 is a rich nearby cluster at $z=0.0542$ (Struble \& Rood 1999 ) in the stage of a violent merger. It has been actively studied in the optical and X-ray bands, and is considered the prototype of a major cluster merger. Previous studies revealed that the cluster has a complex galaxy distribution (Fabricant et al. 1986, Zabludoff \& Zaritzki 1995), X-ray morphology, and gas temperature structure (Henry \& Briel 1995; Henrikesen \& Markevitch 1996; Markevitch et al. 2003, hereafter M03; Henry et al. 2004). These data indicate that A754 is undergoing a major merger of two components along an east-west axis, probably with a non-zero impact parameter. Hydrodynamic simulations were able to reproduce most of the observed X-ray features in A754 by considering an offaxis collision between two subclusters with a mass ratio of 2.5:1 (Roettiger et al. 1998). Chandra data suggested that the merger may be more complex, possibly involving a third subcluster or a cloud of cool gas decoupled from its former host subcluster (M03).

Krivonos et al. (2003) reported an edge-like surface brightness feature in the ROSAT PSPC image of the cluster, located east of the core, which looked like a shock front. They modeled it with a radial density profile with a jump, deriving a Mach number $M=1.71_{-0.24}^{+0.45}$ from this jump under the assump- 
tion that this is indeed a shock front. This brightness feature was also apparent in the Chandra image by M03, but it was at the edge of the Chandra field of view, which did not allow its detailed study. Henry et al. (2004) presented a temperature map of the cluster from an XMM-Newton observation. The presumed post-shock region was shown to be hot, as expected for a shock front. However, because the X-ray brightness ahead of this front is very low, the $X M M$ observation did not have sufficient sensitivity to determine the temperature in the pre-shock region, in order to detect the expected temperature jump. They were only able to derive a temperature in the annulus around the cluster that included the pre-shock region (region 12 in Fig. 6 of Henry et al. 2004), but that annulus is dominated by emission from regions on the other side of the cluster, unrelated to the shock front. We note that many features that look like shocks in the X-ray images turned out to be "cold fronts" (Markevitch \& Vikhlinin 2007), and the difference between these phenomena is the sign of the temperature jump at the feature. In this work, we present a direct temperature measurement in the pre-shock region from a new Chandra observation.

A754 also exhibits evidence for ultrarelativistic electrons and magnetic fields that coexist with the ICM. Fusco-Femiano et al. (2003) reported a $3 \sigma$ hard X-ray excess at $E>45$ $\mathrm{keV}$ with BeppoSAX, which may come from inverse Compton scattering of the Cosmic Microwave Background (CMB) photons on relativistic electrons. A clear evidence of nonthermal emission in $\mathrm{A} 754$ comes from the radio observations. A radio halo was detected at $74 \mathrm{MHz}, 330 \mathrm{MHz}$ and $1.4 \mathrm{GHz}$ with the VLA (Kassim et al. 2001, hereafter K01; Bacchi et al. 2003, hereafter B03, and references therein). Moreover, the presence of two peripheral radio relics were reported in K01, East and West of the radio halo. Only the eastern emission was seen in B03. The whole cluster diffuse radio emission has been recently studied in the frequency range $150-1360 \mathrm{MHz}$ by Kale et al. (2009).

\section{CHANDRA OBSERVATIONS AND THE SHOCK FRONT}

A754 was first observed with Chandra ACIS-I in October 1999 (OBSID 577). This pointing was centered on the cluster center and used by M03 to study the cluster temperature structure. In Febraury 2009, a new long Chandra ACIS-I observation (OBSID 10743) was pointed on the putative shock front, in order to derive the temperature profile across the shock. The new exposure partially overlaps the old one (see Fig. 1), and in this paper we use both observations for the spectral analysis of regions where they overlap.

Both datasets were processed and cleaned in a standard manner (e.g., Vikhlinin et al. 2005). The data were cleaned of flares and the blank-sky datasets were normalized as described in Markevitch et al. (2003b). OBSID 577 had small flare contamination and the final clean exposure was $39 \mathrm{ks}$. No background flares were present in the new observation, so we used the full exposure of $95 \mathrm{ks}$. The old observation was taken in FAINT mode, while the new one in VFAINT, which allowed additional background filtering to be applied. The detector + sky background was modeled using the blanksky background datasets corresponding to the dates of observations (periods B and E, respectively). We normalized the blank-sky backgrounds using the the ratio of counts in the high-energy band 9.5-12 keV, which is free from the sky emission. This correction was within $10 \%$ of the exposure ratios, as expected.
All point sources were masked out for the extraction of brightness profiles and spectra. The instrument responses for spectral analysis were generated weighting the detector ARF and RMF with the cluster brightness within each spectral extraction region (Vikhlinin et al. 2005). We used the most recent calibration products - namely, version N0008 for the telescope effective area, N0006 for the CCD quantum efficiency and gain files, and N0005 for the ACIS time-dependent low-energy contaminant model. We also assessed the systematic uncertainty of our results by using a newer, experimental update to the ACIS contaminant model (A. Vikhlinin, private communication; see below).

Figure 1 shows a slightly smoothed ACIS image of the cluster in the $0.5-4 \mathrm{keV}$ band, obtained by co-adding the two observations and correcting for exposure nonuniformity. As found by previous studies, the cluster undergoes a complex merger with the main axis along the NW-SE direction. The image clearly shows a brightness edge to the east of the dense elongated core, perpendicular to the merger direction. This is the putative shock front reported by Krivonos et al. (2003). The new observation provides sufficient statistics to determine the exact nature of this feature.

\subsection{Density profile across the edge}

Figure 2 shows a radial X-ray surface brightness profile across this edge, extracted in a $25^{\circ}$ sector shown in Fig. 1 . To facilitate proper geometric modeling, the sector is centered on the center of curvature of the brightness edge (dashed white circle in Fig. 1) and encompasses its most prominent segment (avoiding the apparent decrease of the edge brightness contrast at greater opening angles). All point sources are carefully masked. The energy band for the profile was restricted to $0.5-4 \mathrm{keV}$ to minimize the dependence of X-ray emissivity on temperature and maximize the signal-to-noise ratio. Only OBSID 10743 is used for the brightness profile, since the earlier observation does not cover the pre-shock region. The exposure of this observation alone is more than sufficient for the post-shock region. We consider only the region outside the bright elongated core, which is obviously unrelated to the shock.

The brightness profile across the edge (Fig. 2a) exhibits the typical shape of a projected spherical density discontinuity (Markevitch et al. 2000), and we fit it with such a model, under the assumption that the curvature along the line of sight is the same as in the image plane. The model radial density profile that we use consists of two power laws, $\rho \propto r^{\alpha_{1}}$ and $\rho \propto r^{\alpha_{2}}$, on two sides of the edge, with an abrupt jump at the edge (Fig. 2p). For each set of the profile parameters, we projected the corresponding emission measure profile onto the image plane and fit to the observed X-ray brightness profile (Fig. 21 ), treating the slopes and the radius and amplitude of the jump as free parameters. The fit was restricted to the interesting radial range — staying as close as possible to the edge on the inside in order to avoid contamination from unrelated core structure, but extending as far as possible on the outside for proper deprojection (see dashed ticks on the outer sides of the sector in Fig. 11. As seen from Fig. 2a, the model fits very well. The best-fit density jump, after a 3\% correction of the $0.5-4 \mathrm{keV}$ Chandra brightness for the measured temperature difference across the edge (see $\S 3.2$ below), is $\rho_{2} / \rho_{1}=1.80_{-0.15}^{+0.20}$. The confidence interval is evaluated by allowing all other model parameters to be free.

\subsection{Temperature profile across the edge}




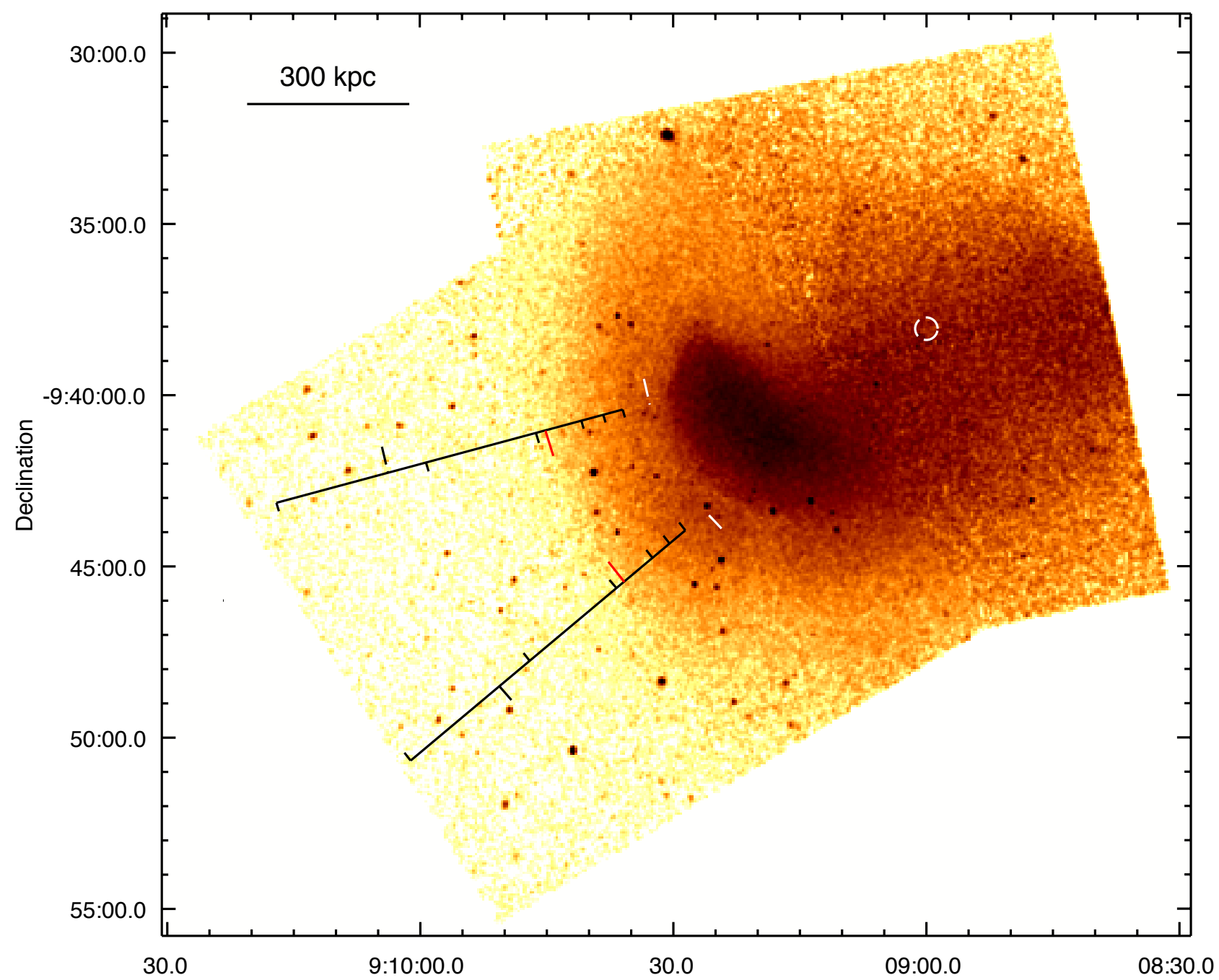

Right ascension

FIG. 1. - Chandra slightly smoothed 0.5-4 keV image of A754 from a sum of the new 95 ks exposure and the archival 39 ks observation. Also shown is the $25^{\circ}$ sector used to extract the X-ray surface brightness profile and for the spectral analysis (see $\S 3.1$ and $\S 3.2$ ). Dashed ticks outside the sector mark the radial range used for the profile fitting; small ticks inside the sector mark the radial bins chosen to derive the temperature profile; bigger red ticks indicate the best-fit density jump, measured from its center of curvature (marked by the dashed circle).

TABLE 1

X-RAY TEMPERATURE FITS

\begin{tabular}{lccc}
\hline \hline Region & obsid 577 & obsid 10743 & Simult. fit \\
\hline \multicolumn{4}{c}{ Current (N0005) ACIS contaminant model: } \\
$1 \ldots \ldots \ldots \ldots$ & $17.6_{-4.4}^{+6.6}$ & $9.0_{-0.7}^{+1.0}$ & $10.1_{-0.9}^{+1.0}$ \\
$2 \ldots \ldots \ldots \ldots$ & $9.6_{-1.7}^{+2.2}$ & $14.2_{-2.1}^{+2.5}$ & $12.5_{-1.8}^{+1.9}$ \\
$3 \ldots \ldots \ldots$ & $8.7_{-1.5}^{+2.2}$ & $16.4_{-2.8}^{+3.7}$ & $13.3_{-1.9}^{+2.5}$ \\
$4 \ldots \ldots \ldots \ldots$ & $\ldots$ & $7.4_{-2.0}^{+2.3}$ & $\ldots$ \\
$5 \ldots \ldots \ldots$ & $\ldots$ & $4.5_{-1.5}^{+2.4}$ & $\ldots$ \\
3 deproj $\ldots \ldots$ & $\ldots$ & $\ldots$ & $16.4_{-3.5}^{+5.1}$ \\
\hline \multicolumn{5}{c}{ Experimental ACIS contaminant model: } \\
$3 \ldots \ldots \ldots$ & $8.7_{-1.5}^{+2.2}$ & $14.2_{-2.4}^{+2.8}$ & $12.2_{-1.8}^{+2.1}$ \\
$4 \ldots \ldots \ldots$ & $\ldots$ & $6.6_{-1.5}^{+2.3}$ & $\ldots$ \\
\hline
\end{tabular}

We now extract spectra from several radial bins in the same sector across the density edge (Fig $1, \$ 3.1$ ) to determine the gas temperature jump across the edge. There is enough statistical accuracy to divide the low-brightness region outside the edge into two bins, and the inner brighter region into three bins, avoiding getting to close to the cluster cool elongated core. The five radial bins are marked by ticks inside the sector (Fig 1 ; ; the longer red ticks mark the best-fit position of the density jump (Fig 1 , \$3.1). The first high-brightness region adjacent to the jump extends across the best-fit jump position a bit, in order to avoid contaminating the adjacent lowbrightness region by any irregularities in the edge shape. The spectra were fit in the $0.8-9 \mathrm{keV}$ band with XSPEC, using the absorbed thermal plasma model WABS(APEC), with elemental abundances as free parameters, fixing the absorption column to the Galactic value $\left(N_{H}=4.82 \times 10^{20} \mathrm{~cm}^{-2}\right.$, Kalberla et al. 2005). We also tried to free $N_{H}$, and all fits were consistent with the Galactic value. All fits had acceptable $\chi^{2}$ values. The systematic uncertainty of background modeling was evaluated by varying the blank-sky background normalization by $\pm 2 \%$ (68\%, Hickox \& Markevitch 2005) and added in quadrature to the statistical uncertainties. It was negligible for the brighter 

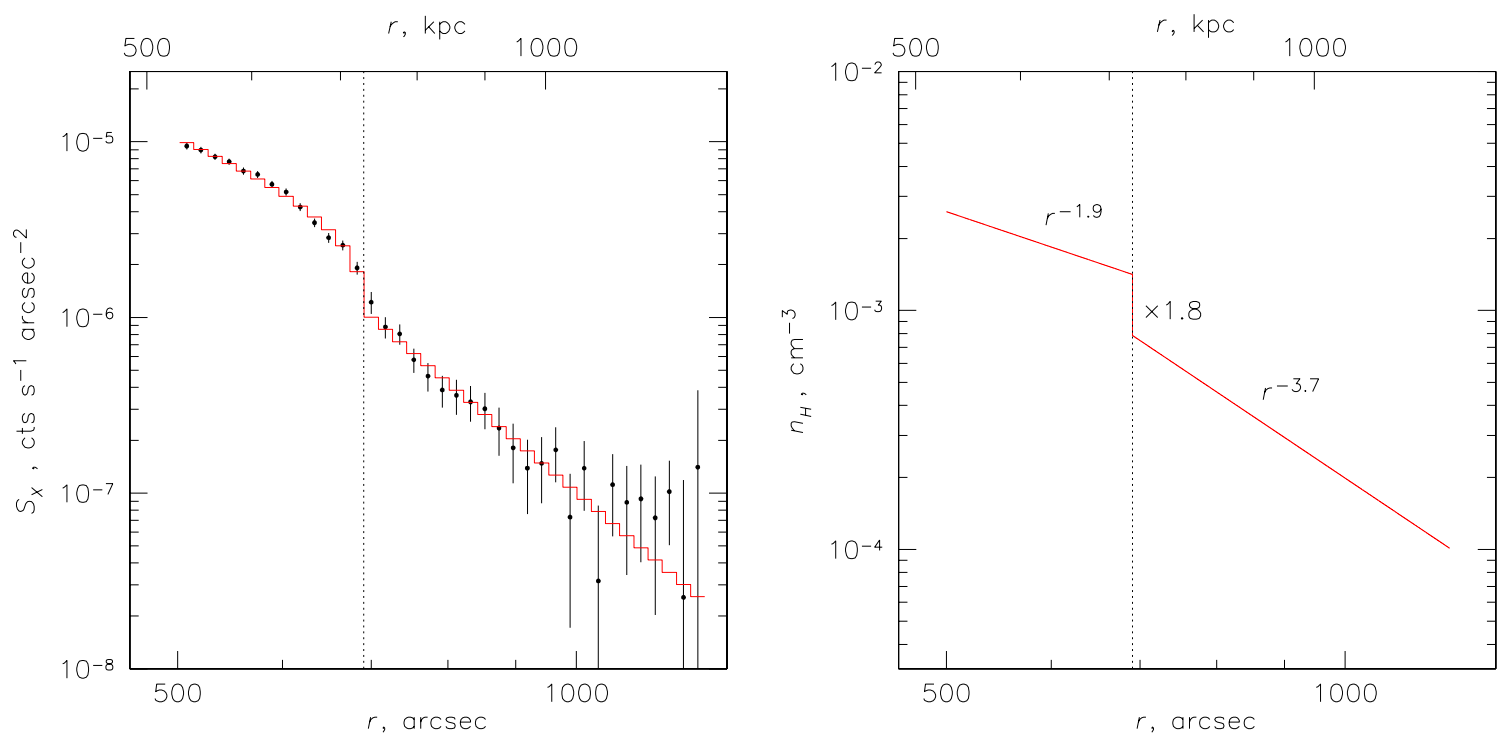

FIG. 2.- (a) The 0.5-4 keV surface brightness profile across the front in the sector shown in Fig. 11 (see text). Errors are $1 \sigma$; histogram shows the best-fit model that corresponds to the radial density profile shown in panel $(b)$. The dashed line marks the best-fit position of the shock front (measured from its center of curvature).

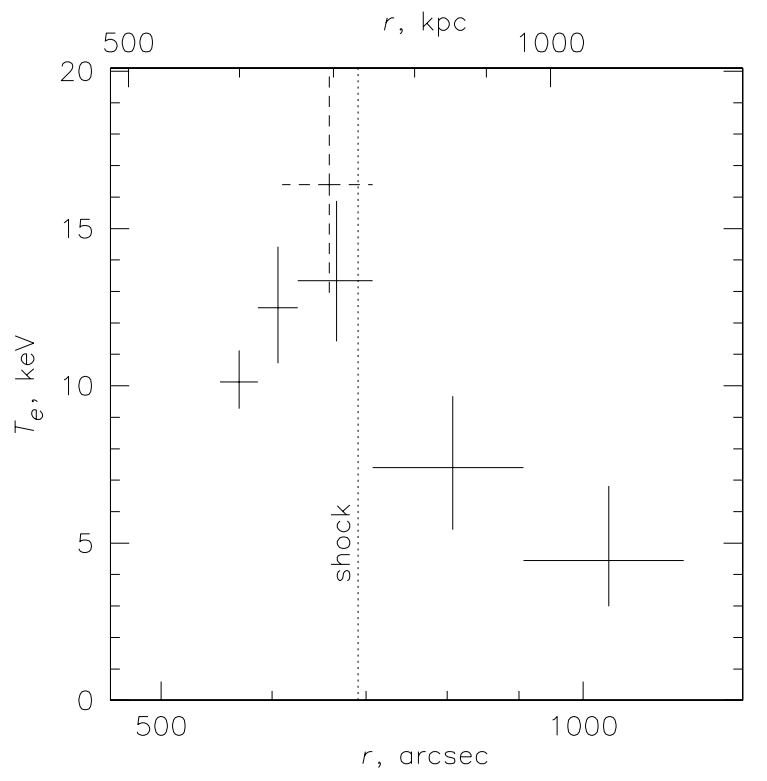

FIG. 3.- Temperature profile across the shock (errors are 68\%). Solid crosses show projected temperature fits; dashed line for the first post-shock region shows a deprojected temperature (modeling the projected contribution of the pre-shock gas based on the best-fit density model). For post-shock regions, simultaneous fits for two observations are shown, while two outer regions are covered only by the recent observation (see Table 1 for more details). Dotted vertical line shows the position of the shock front from the density fit (see Fig.2).

regions inside the edge, but similar to the statistical uncertainty for the two low-brightness bins (the observation exposure was selected to achieve this).

Solid crosses in Fig. 3 show the resulting temperature values, using the simultaneous fit to both observations for the inner regions and OBSID 10743 for the outer regions. The figure shows a clear temperature jump of the sign that corresponds to a shock front.

Table 1 gives separate fits for two pointings; the regions are numbered in order of increasing radius in Fig. 3 We note that two Chandra observations are in mild disagreement for the post-shock regions (though none deviates by more than $2 \sigma$ from the simultaneous fit). This may be caused by residual calibration problems - these regions are at the edge of the ACIS-I field of view in OBSID 577, while at the center in OBSID 10743. In addition, these observations are separated by 10 years. We tried an experimental update to the recently released time dependence of the time-dependent ACIS-I contaminant model (A. Vikhlinin, private communication) to see if it changes the results qualitatively, for the two bins adjacent to the front. The results are shown in Table 1 . The disagreement is slightly reduced. Most importantly, the temperature jump does not change qualitatively, and is also seen in the recent observation alone, using either calibration. To the extent the results from different spatial regions can be compared, our post-shock high temperatures are in broad agreement with those derived with $X M M$ by Henry et al. (2004).

Thus, the temperature profile confirms that the brightness edge is a shock front. From the Rankine-Hugoniot jump conditions, we can use our best-fit gas density jump to derive a Mach number of this shock. Assuming monoatomic gas with $\gamma=5 / 3$, we obtain $M=1.57_{-0.12}^{+0.16}$

The temperature jump can give an independent Mach number estimate, though usually with a lower accuracy. To check for the consistency of these estimates, we first get a deprojected temperature in the first post-shock radial bin, since in the spherical geometry, the temperature we measure there is affected by projection of the cooler emission from the outer, pre-shock bins. (At the same time, the pre-shock temperature profile appears consistent with isothermal, so the true temperature should be close to the projected one, to a sufficient accuracy). From the best-fit density model, we estimate a projected emission measure fraction in bin 3 from the outer regions 4 and 5 in Fig. 3 to be $21 \%$ and 3\%, respectively. $\mathrm{Ne}$ glecting the latter and fitting the spectrum for region 3 using an additional thermal model with the normalization and temperature (including its uncertainties) corresponding to region 4 , we obtain a "deprojected" temperature shown by the dashed 
cross in Fig. 3 .

The ratio of the post-shock to pre-shock temperatures is thus $2.2_{-0.7}^{+1.1}$, which corresponds to $M=2.1_{-0.6}^{+0.7}(68 \%)$, consistent (within its expected larger uncertainties) with the value from the density jump obtained above.

\section{RADIO OBSERVATIONS}

In this section, we present our GMRT observations of A754 at $330 \mathrm{MHz}$, and analysis of the archival $V L A$ data at $1.4 \mathrm{GHz}$. Details of observations are summarized in Table 2, which reports the telescope, project code, observing date, frequency, total bandwidth, total time on source, synthesized half-power bandwidth (HPBW) of the full array, rms level $(1 \sigma)$ at full resolution, $u-v$ range, and the largest detectable structure (LDS).

\subsection{GMRT observations at $325 \mathrm{MHz}$}

A754 was observed with GMRT at $325 \mathrm{MHz}$ in June 2005 for a total time on source of $2.5 \mathrm{hr}$ (Table 2). The observations were carried out using the upper and lower side bands simultaneously (USB and LSB, respectively), for a total observing bandwidth of $32 \mathrm{MHz}$. The default spectral-line observing mode was used, with 128 channels for each band and a spectral resolution of $125 \mathrm{kHz} /$ channel. The LSB dataset was corrupted and could not be processed, so only the USB data, which are centered at approximately $330 \mathrm{MHz}$, were used to produce the images presented here. The dataset was calibrated and analyzed using the NRAO Astronomical Image Processing System package (AIPS). We refer to Giacintucci et al. (2008) for a complete description of the data reduction procedure used in this paper.

Due to the large field of view of GMRT at $330 \mathrm{MHz}$ (primary beam $\sim 1.8^{\circ}$ ), we used the wide-field imaging technique at each step of the phase self-calibration process, to account for the non-planar nature of the sky. We covered a field of $\sim 2.7^{\circ} \times 2.7^{\circ}$ with 25 facets. The final images were produced using the multi-scale CLEAN implemented in the AIPS task IMAGR, which results in better imaging of extended sources compared to the traditional CLEAN (e.g., Clarke \& Ensslin 2006; for a detailed discussion, see Appendix A in Greisen, Spekkens, \& van Moorsel 2009). We used three circular Gaussians as model components. One of the Gaussian was chosen to have zero width to accurately model point sources and small-scale structures, and the other two have a width of $20^{\prime \prime}$ and $45^{\prime \prime}$ respectively, to progressively highlight the extended emission during the clean.

Beyond the image at full resolution $\left(10.0^{\prime \prime} \times 9.1^{\prime \prime}\right)$, we produced images with lower resolution (down to $\sim 100^{\prime \prime}$ ), tapering the $u-v$ data by means of the parameters robust and uvtaper in the task IMAGR. Even though only half of the data was usable, the sensitivity of the final images is quite good - the rms noise level $(1 \sigma)$ ranges from $0.45 \mathrm{mJy}^{\text {beam }}{ }^{-1}$ in the full resolution image to $\sim 1 \mathrm{mJy}^{\text {beam }^{-1}}$ in the lowest resolution images. We estimate that the flux density calibration uncertainties are within $5 \%$.

\subsection{VLA archive data at $1.4 \mathrm{GHz}$}

VLA observations of A754 at $1.4 \mathrm{GHz}$ (project AF372) were presented by $\mathrm{B} 03$. We extracted these observations from the archive and re-analyzed them in order to ensure the best possible comparison with our GMRT data. The observations were obtained using the D configuration and two IFs, centered at $1365 \mathrm{MHz}$ and $1435 \mathrm{MHz}$ (see Table 2 for details on the observations). Standard calibration and imaging were carried out using AIPS. The dataset was self-calibrated in phase only. The higher frequency IF was found to be affected by strong radio interference, which compromises the quality of the images produced using both IFs. For this reason, only the lowfrequency IF was used for the analysis presented in this paper. The final images were obtained implementing the multi-scale clean option in IMAGR, as for the $330 \mathrm{MHz}$ data (see $\$ 4.1$ ). The rms noise in the image at full resolution is $50 \mu \mathrm{Jy}$ beam $^{-1}$ (Table 2). A slightly higher noise $\left(1 \sigma \sim 60-70 \mu \mathrm{Jy}^{\text {beam }}{ }^{-1}\right.$ was achieved in the low-resolution (HPBW $\sim 70^{\prime \prime}$ ) images. The average residual amplitude errors in the data are of the order of $\lesssim 5 \%$.

\section{RADIO ANALYSIS}

In Fig. 4, we present the new ${ }^{3}$ GMRT image at $330 \mathrm{MHz}$ at the resolution of $63^{\prime \prime} \times 52^{\prime \prime}$ (left panel) and the VLA 1365 $\mathrm{MHz}$ image at full resolution $\left(\mathrm{HPBW}=64^{\prime \prime} \times 39^{\prime \prime}\right)$, overlaid on the optical POSS-2 red frame of A 754 (right panel). The first positive contour corresponds to the $3 \sigma$ level in both images (i.e., $3.3 \mathrm{mJy}^{\text {beam }}{ }^{-1}$ at $330 \mathrm{MHz}$ and $0.21 \mathrm{mJy}^{\text {beam }}{ }^{-1}$ at $1365 \mathrm{MHz}$ ). Numerous discrete radio sources are located in the cluster area. Some of these sources are embedded within the diffuse emission. We identify all the radio galaxies found by B03. Following their same notation, these are labelled from $\mathrm{S} 1$ to $\mathrm{S} 8$ in both images. The point source S5 is undetected at $330 \mathrm{MHz}$.

In order to properly image the cluster diffuse emission, we subtracted all the discrete radio galaxies in the cluster region from the $u-v$ data at both frequencies. We first subtracted the brightest radio sources in the field (including the extended radio galaxy north-east of S1 in Fig. 4), to simplify the detection and subtraction of the fainter discrete galaxies embedded in the diffuse emission (from S1 to S8).

We finally produced a set of low-resolution images from the "subtracted" data sets at both frequencies, to highlight the extended emission only.

Our results are shown in Fig. 5. The left panel shows contours of our VLA low-resolution image at $1365 \mathrm{MHz}$ $\left(\mathrm{HPBW}=70^{\prime \prime} \times 70^{\prime \prime}\right)$, before the subtraction of discrete radio sources ${ }^{4}$ The rms noise of the image is $60 \mu \mathrm{Jy} \mathrm{beam}^{-1}(1$ $\sigma)$; this is significantly lower with respect to the noise level reached by B03 in their image of same resolution (see Fig. 3 of B03). In the right panel of Fig. 5 we present images of the diffuse emission only, obtained after the subtraction of discrete sources described above. The GMRT $330 \mathrm{MHz}$ intensity contours at the resolution of $109^{\prime \prime} \times 74^{\prime \prime}$ are overlaid on the $V L A 1365 \mathrm{MHz}$ image (grayscale), produced with a restoring beam of $70^{\prime \prime} \times 70^{\prime \prime}$. Both images show the very large extent and complexity of the diffuse emission in A754. The diffuse source spans $\sim 1.4 \mathrm{Mpc}$ in the east-west direction and $\sim 750$ $\mathrm{kpc}$ along the north-south axis, as measured from the $3 \sigma$ contour level at $330 \mathrm{MHz}$. The $1365 \mathrm{MHz}$ image reveal a ridge in the radio brightness, extended westbound the $330 \mathrm{MHz}$ contours. On the other hand, the high frequency emission is less extended in the eastern region (see below). We point out that these are reliable features, not due to a shift between the two images. Indeed we carefully checked the astrometry of our

\footnotetext{
3 These observations, available from the GMRT public archive, has been also recently used by Kale et al. 2009, for their low frequency study of A 754 However, they do not show the radio images obtained from these data.

4 Here S9 ? indicate the peak of emission visible to the north-east of S7 in Fig. 4 This apparently coincides with an optical counterpart, and might be an additional point source; however we considered it as a peak of the diffuse emission and we did not subtract it from the data.
} 
TABLE 2

SUMMARY OF THE RADIO OBSERVATIONS.

\begin{tabular}{|c|c|c|c|c|c|c|c|c|c|}
\hline $\begin{array}{c}\text { Radio } \\
\text { telescope }\end{array}$ & $\begin{array}{l}\text { Project } \\
\text { code }\end{array}$ & $\begin{array}{c}\text { Observation } \\
\text { date }\end{array}$ & $\begin{array}{c}\nu \\
(\mathrm{MHz})\end{array}$ & $\begin{array}{l}\Delta \nu \\
(\mathrm{MHz})\end{array}$ & $\begin{array}{c}\mathrm{t} \\
(\mathrm{min})\end{array}$ & $\begin{array}{l}\text { HPBW, p.a. } \\
\left({ }^{\prime \prime} \times{ }^{\prime \prime},{ }^{\circ}\right)\end{array}$ & $\begin{array}{c}\text { rms } \\
\left(\mu \mathrm{Jy} \mathrm{beam}^{-1}\right)\end{array}$ & $\begin{array}{c}u-v \text { range } \\
(\mathrm{k} \lambda)\end{array}$ & $\begin{array}{l}\text { LDS } \\
\left(^{\prime}\right)\end{array}$ \\
\hline$G M R T$ & 08GBA01 & June 23, 2005 & 325 & $32(16)^{*}$ & 150 & $10.0 \times 9.1,-64$ & 450 & $\sim 0.08-25$ & $\sim 32$ \\
\hline$V L A-\mathrm{D}$ & AF372 & Sept. 25,2000 & $1365 / 1435^{* *}$ & 50 & 160 & $64.5 \times 38.8,5$ & 50 & $\sim 0.13-4.7$ & $\sim 15$ \\
\hline
\end{tabular}

Notes to Table 2 * Only the USB dataset was used for the analysis (see Sect. 4.1. ** Only the the lower frequency IF was used for the analysis (see Sect. 4.2.
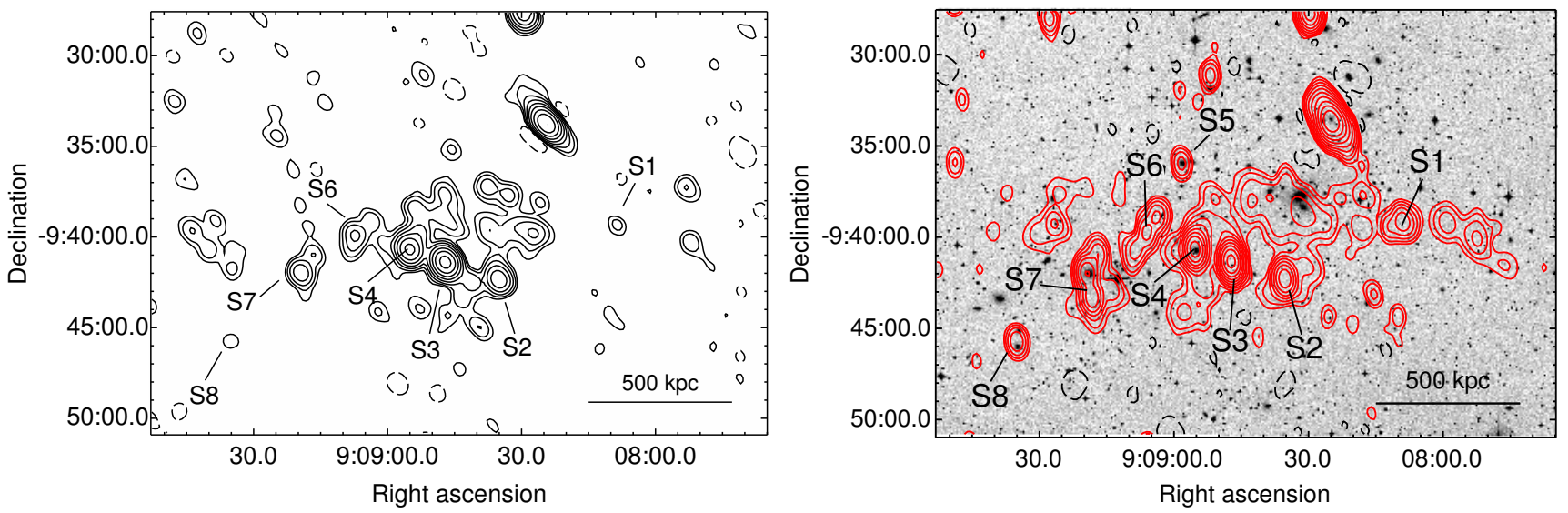

FIG. 4.- Left: GMRT $330 \mathrm{MHz}$ image of A 754 at the resolution of $63.5^{\prime \prime} \times 52.1^{\prime \prime}$, p.a. $30^{\circ}$. The $1 \sigma$ level is $1.1 \mathrm{mJy}$ beam ${ }^{-1}$. Contours start at $-3.3 \mathrm{mJy}$ beam $^{-1}$ (dashed) and $+3.3 \mathrm{mJy}^{\text {beam }}{ }^{-1}$, and then scale by a factor of 2. Right: VLA full-resolution image at $1365 \mathrm{MHz}$ (contours), overlaid on the optical POSS-2 red image of A 754 . The restoring beam is $64.5^{\prime \prime} \times 38.8^{\prime \prime}$, p.a. $5^{\circ}$, and the $1 \sigma$ noise level is $50 \mu \mathrm{Jy} \mathrm{beam}^{-1}$. Contours are spaced by a factor of 2 , starting from $\pm 0.15 \mathrm{mJy} \mathrm{beam}^{-1}$ (negative contours are shown as dashed). In both images, labels indicate the discrete radio galaxies identified at $1.4 \mathrm{GHz}$ by B03.
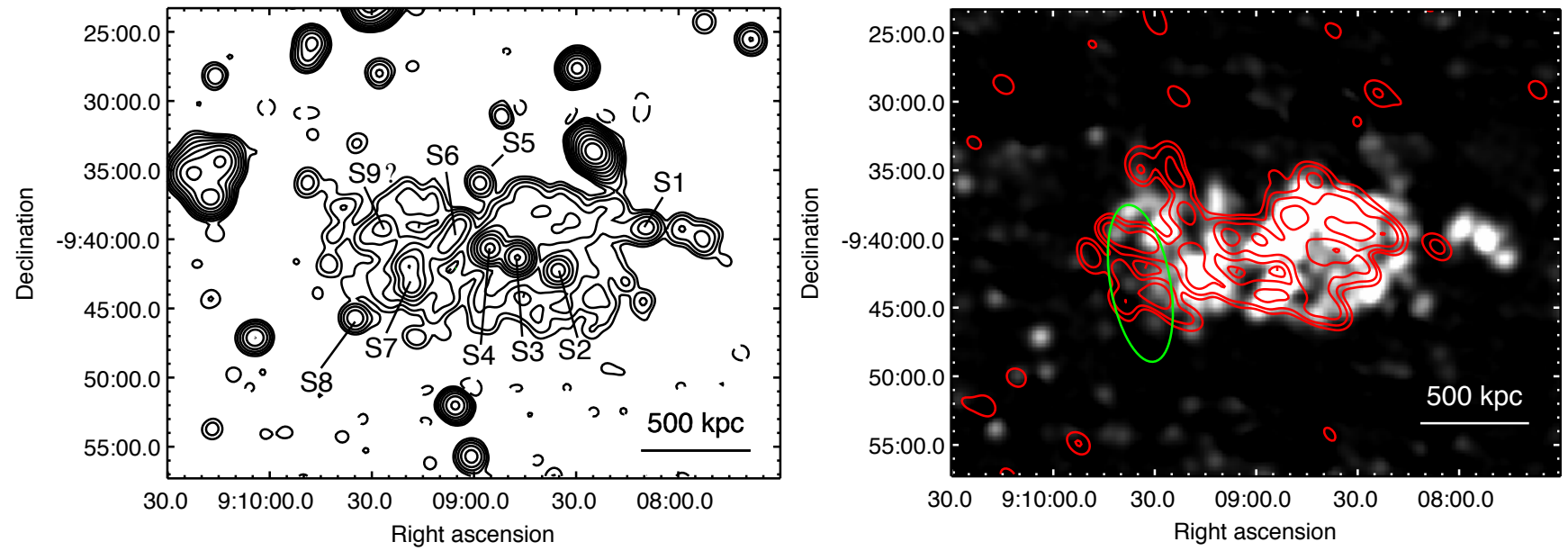

FIG. 5.- Left: VLA low-resolution image at $1365 \mathrm{MHz}$. The restoring beam is $70^{\prime \prime} \times 70^{\prime \prime}$, p.a. 0 ; contours are spaced by a factor 2 starting from $\pm 3 \sigma$, the $1 \sigma$ level is $60 \mu \mathrm{Jy}_{\text {beam }^{-1}}$. Labels indicate the discrete radio galaxies identified within the diffuse emission. Negative contours are shown as dashed. Ticks mark contours that correspond to holes in the radio brightness. Right: diffuse emission after the subtraction of all identifiable discrete sources. Red contours show a low-resolution GMRT image at $330 \mathrm{MHz}$ with a restoring beam of $109^{\prime \prime} \times 74^{\prime \prime}$, p.a. $43^{\circ}$ and the $1 \sigma$ noise level of $1 \mathrm{mJy}$ beam ${ }^{-1}$. Contours are spaced by a factor of 2, starting from $3 \sigma$. Greyscale shows a low-resolution $V L A$ image at $1365 \mathrm{MHz}-$ same as in left panel but without the discrete sources. The peak flux density is $3.77 \mathrm{mJy}$ beam $^{-1}$ and the lowest level of greyscale corresponds to $\sim 2 \sigma$. The green ellipse is the area covered by the $2 \sigma$ contour $\left(0.4\right.$ Jy beam $\left.{ }^{-1}\right)$ of the radio relic in the $V L A$ image at $74 \mathrm{MHz}(\mathrm{K} 01)$. Ticks mark contours that correspond to holes in the radio brightness. 


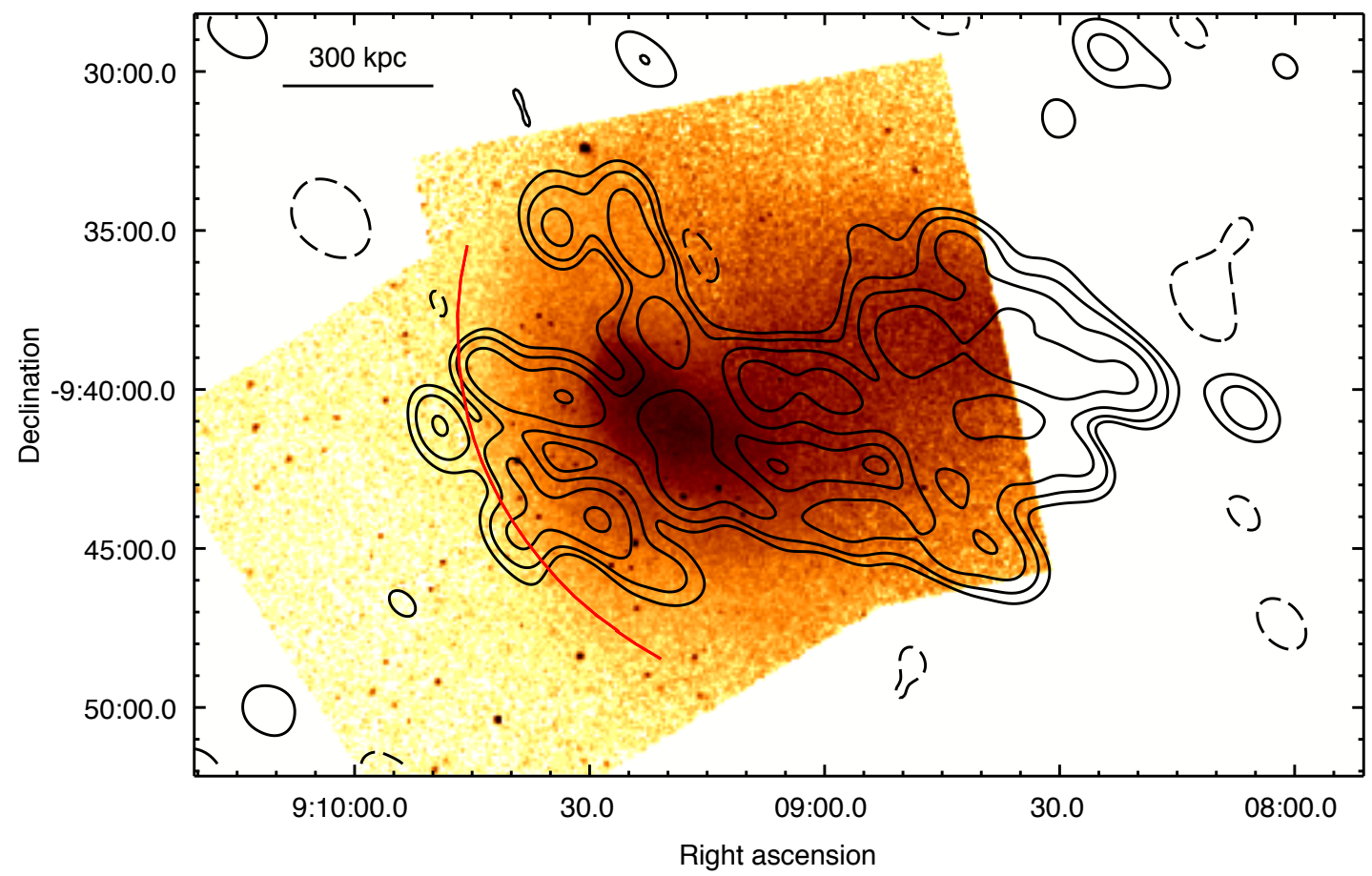

FIG. 6. - GMRT low resolution radio contours (same as in Fig. 5 right panel), overlaid on the Chandra image of A754 (same as Fig. 1 see $\$ 3$. The dashed contours show the $3 \sigma$ negative values; the red arc indicates the best fit position of the density jump (see $\$ 3.1$. 
radio images at both frequencies, by comparing the positions of the peaks of the radio sources with that of the optical counterparts.

The green ellipse in Fig. 5 (right panel) represents the $2 \sigma$ contour $\left(0.4 \mathrm{Jy} \mathrm{beam}^{-1}\right)$ of the eastern radio relic found in the $74 \mathrm{MHz} V L A$ image by $\mathrm{K} 01$. The $330 \mathrm{MHz}$ diffuse emission extends eastward to the outer side of this relic. Interestingly, we notice that the shock front found in $\$ 3.2$ coincides with the edge of the emission at $330 \mathrm{MHz}$ (see Fig. 6), possibly suggesting a connection between the radio edge and the shock (see $\$ 6$ )

We detect significant emission at $1365 \mathrm{MHz}$ only in part of the region covered by the green ellipse (see Fig. 5). Our VLA images at $1365 \mathrm{MHz}$ show more diffuse emission, south of the S2-S4 sources, with respect to B03. This is most likely due to differences in the editing of the data, in the imaging procedure (multi-scale clean, see $\$ 4.2$ ) and in the accuracy of the subtraction of discrete sources (see also the comment in B03); our final rms value is indeed a factor 1.6 better.

We do not detect any radio emission in the region of the western relic found by $\mathrm{K} 01$.

\subsection{Spectrum of the diffuse radio emission}

The radio images in Fig. 5 (right panel) show a complex, Mpc-scale diffuse source associated with A754. We use our $330 \mathrm{MHz}$ GMRT and the reprocessed $1365 \mathrm{MHz} V L A$ data presented in $\$ 4$, along with the VLA image at $74 \mathrm{MHz}$ kindly provided by N. Kassim (see Fig. 1 in K01), to derive its integrated spectrum. The flux densities of the diffuse emission, after the subtraction of the discrete sources, are $828 \pm 41$ $\mathrm{mJy}$ and $89 \pm 5 \mathrm{mJy}$ at $330 \mathrm{MHz}$ and $1365 \mathrm{MHz}$, respectively. These are also reported in Table 3, along with the associated uncertainties and angular resolution of the images used for the measurements. We obtained these values by integrating over the same area that encompasses the whole diffuse emission as detected at both frequencies ${ }^{5}$. We note that the flux density at $1365 \mathrm{MHz}$ is lower than the value reported in B03 where, however, the authors notice that the subtraction of discrete sources is not accurate (B03; see also $\S 5$. The flux density of the whole diffuse emission at $74 \mathrm{MHz}$ is $\sim 6.6 \mathrm{Jy}$ (Tab. 3). This value was integrated over the same area described above and does not include the contribution of the brightest radio galaxies at the cluster center (i.e., S2, S3 and $\mathrm{S} 4$, and the extended radio galaxy north-east of $\mathrm{S} 1$; see Fig. 4) which amounts to a total of $\sim 2.9 \mathrm{Jy}$ (as estimated in K01). However, due to the lower sensitivity, only part of the extended emission visible at $330 \mathrm{MHz}$ and $1365 \mathrm{MHz}$ is detected at $74 \mathrm{MHz}$. Thus, our estimate should be considered as a lower limit to the flux. The spectral index for the whole diffuse emission between 330 and $1365 \mathrm{MHz}$ is $\alpha=1.57 \pm 0.05$. This value is steeper than typically found for giant radio halos ( $\alpha \sim 1.2-1.3$; e.g., Ferrari et al. 2008), although a number of halos with ultra-steep spectrum $(\alpha>1.5)$ has been discovered in the past few years (e.g., Brunetti et al. 2008, Brentjens 2008, Macario et al. 2010).

For simplicity, in the following we will call relic the feature discovered by $\mathrm{K} 01$ at $74 \mathrm{MHz}$, that they defined as eastern radio relic (see also discussion, $\$ 6$.

In our GMRT image at $330 \mathrm{MHz}$, the relic does not appear as a distinct feature separated from the radio halo, nor a clear increment of the surface brightness of the diffuse emission is

\footnotetext{
${ }^{5}$ We also checked that no significant change in the spectral index occurs by integrating within the emission at the $2 \sigma$ level.
}

detected in that region. This may imply that the spatial segregation between the relic and the halo in the $74 \mathrm{MHz}$ image (see Fig. 1 in K01) may be due to a steeper spectrum of the relic at $\nu \lesssim 300 \mathrm{MHz}$ combined with the lower sensitivity of the $74 \mathrm{MHz}$ observation.

To obtain the radio spectrum of the relic region, we integrated the flux densities at $330 \mathrm{MHz}$ and $1365 \mathrm{MHz}$ over the area defined by the $2 \sigma$ contour level of the relic at $74 \mathrm{MHz}$ (green ellipse in Fig. 5 right panel). We obtained $106 \pm 5 \mathrm{mJy}$ at 330 $\mathrm{MHz}$ and 6.0 $10.3 \mathrm{mJy}$ at $1365 \mathrm{MHz}$ (see Table 3).

In Fig. 7 we show the integrated radio spectrum of the relic, in the frequency range $74-1365 \mathrm{MHz}$. The relic has indeed a very steep spectrum, with $\alpha_{0.07 G H z}^{0.3 G H z}=1.77 \pm 0.10$, and $\alpha_{0.3 \mathrm{GHz}}^{1.4 \mathrm{GHz}}=2.02 \pm 0.04$. An extrapolation of this power law to lower frequencies predicts a $74 \mathrm{MHz}$ flux density that is consistent with that reported in K01. For a direct comparison, the flux densities of the whole diffuse emission are also shown.

A spectral study of the diffuse emission in A 754 was presented in Kale et al. (2009). However their analysis is limited to specific regions of emission detected in their GMRT 150 $\mathrm{MHz}$ image (that do not show the whole extended emission). Therefore, we cannot compare our spectral results to those found by them.

TABLE 3

FLUX DENSITIES OF THE DIFFUSE RADIO EMISSION IN A 754

\begin{tabular}{ccccc}
\hline \hline \multirow{2}{*}{$\nu(\mathrm{MHz})$} & $\mathrm{HPBW},{ }^{\prime \prime} \times{ }^{\prime \prime}$ & \multicolumn{2}{c}{$\mathrm{S}_{\nu}(\mathrm{mJy})$} & \multirow{2}{*}{ Ref. } \\
\cline { 3 - 4 } & & relic & whole emission nn & \\
\hline 74 & $316 \times 232$ & $1489 \pm 223$ & $\sim 6600$ & this work; K01 \\
330 & $109 \times 74$ & $106 \pm 5$ & $828 \pm 41$ & this work; Fig. 5 \\
1365 & $70.0 \times 70.0$ & $6.0 \pm 0.3$ & $89 \pm 5$ & this work; Fig. 5 \\
\hline
\end{tabular}

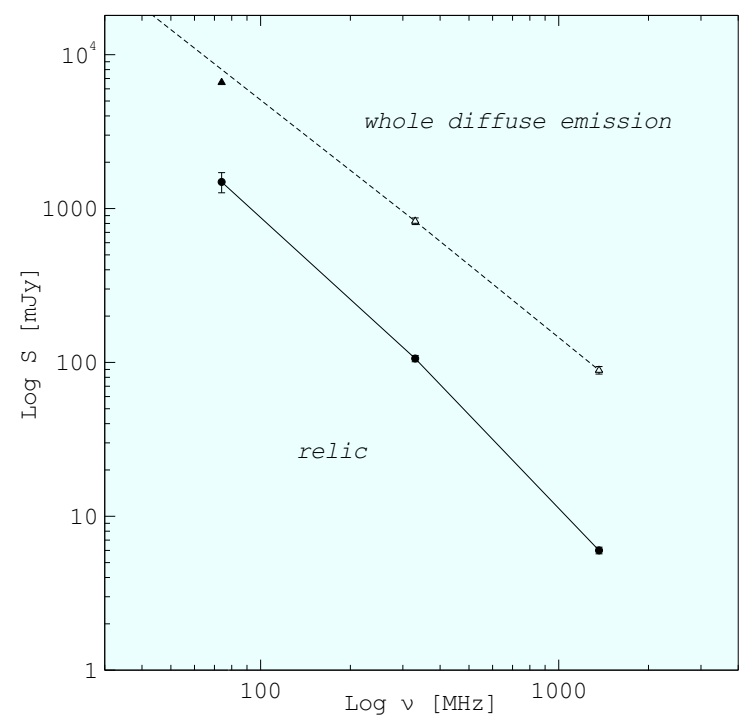

FIG. 7.- Integrated spectrum of relic region (filled dots) in A 754 in the frequency range $74-1365 \mathrm{MHz}$. The solid line connects the values of flux density reported in Table 3 The spectrum of the whole diffuse emission between 330 and $1365 \mathrm{MHz}$ is also shown (open triangles and dashed line); the estimated flux density at $74 \mathrm{MHz}$ (filled triangle) is in reasonable agreement with the extrapolation of the spectrum. 


\section{DISCUSSION}

The main result of the present paper is the clear X-ray detection of a shock front in the intracluster gas of A 754, which coincides with the edge of non-thermal radio diffuse emission at $325 \mathrm{MHz}$.

Particle acceleration at the shock front cannot be responsible for the whole Mpc-scale diffuse emission seen in Fig. 5 Given the shock parameters derived in $\S 3.2$, the downstream velocity of the gas is $\simeq 1100 \mathrm{~km} \mathrm{~s}^{-1}$, which implies that the size of the diffuse emission produced by the shock can be only $\sim 100-200 \mathrm{kpc}$, once the radiative lifetime of the emitting electrons $\left(1-2 \times 10^{8} \mathrm{yr}\right)$ is taken into account (see also the discussion in Markevitch et al. 2005 and Brunetti et al. 2008). However, the spatial coincidence of the ultra-steep spectrum radio edge with the shock front suggests a physical connection between these two features, and possibly an indirect connection between the shock and the diffuse emission on the larger scale. If one assumes that relativistic electrons in the relic region are accelerated via Fermi mechanism by the shock front with $M \sim 1.6$ (as observed in X-rays, see $\$ 3.2$, the expected slope of the radio spectrum from that region, accounting for the downstream losses, is $\alpha \sim 2.3\left(\alpha=\alpha_{i n j}+1 / 2\right.$, where $\alpha_{i n j}=(p-1) / 2$ and $p=2\left(M^{2}+1\right) /\left(M^{2}-1\right)$; e.g., Blandford\& Eichler 1987), in rough agreement with the radio observation (see $\$ 5.1$. However, we can rule out direct shock acceleration on energy grounds. If we extrapolate the power law spectrum of electrons responsible for the relic emission down to thermal energies (as we should if the seed electrons are thermal), the energy in relativistic electrons will be of the same order as the thermal energy in the ICM requiring an implausible high acceleration efficiency. This energy argument implies that observable radio emission cannot be produced at shocks with $M<2$ (Hoeft \& Brrüggen 2007).

Below we discuss possible scenarios for the origin of the cluster-scale diffuse emission in A754.

1. The edge emission might arise from relativistic plasma that is re-energized at the shock. If fossil electrons are present in the ICM, compression by the shock can significantly increase their synchrotron emission at the observing frequency (e.g., Ensslin et al. 1998, Ensslin \& Gopal-Krishna 2001, Markevitch et al 2005). Adiabatic compression increases the maximum synchrotron frequency emitted by a population of fossil electrons by $r^{4 / 3}$ times (e.g., Markevitch et al. 2005), where $r$ is the shock compression factor (considering a shock moving perpendicular to the line of sight). In our case, this implies only a moderate boosting of a factor 2 . The resulting synchrotron emission may light up at our observing frequency. The drawback of this scenario is that in this case the fossil plasma must have been injected in the ICM only a few $10^{8}$ yrs ago, because the fossil electrons must exist at energies just below those necessary to emit at the observed frequency, and at those energies the lifetime is short.

This problem remains even if one assumes that the fossil plasma is confined (not mixed) by the ICM (Ensslin \& Gopal-Krishna 2001), due to the low Mach number of the shock.

2. Shock reacceleration of fossil relativistic plasma can potentially be an efficient process even in the case of weak shocks, though this process at weak shocks is poorly understood and its efficiency is uncertain. As- suming the standard linear shock acceleration theory (e.g., Blandford \& Eichler 1987), the spectrum of reaccelerated electrons is:

$$
N(\gamma)=(p+2) \gamma^{-p} \int_{\gamma_{\min }}^{\gamma} N_{f s s}(x) x^{p-1} d x
$$

where $p=2\left(M^{2}+1\right) /\left(M^{2}-1\right)$ is the slope of the spectrum of accelerated electrons immediately after the shock, $N_{f s s}$ is the spectrum of fossil electrons in the upstream region, and $\gamma_{\min }$ is the minimum energy of the fossil electrons at which the reacceleration process works. If the fossil relativistic electrons have an exponential cutoff in their power spectrum (as they should if they result from radiative cooling, e.g. Sarazin 2002), shock reacceleration should create a spectrum above that cutoff with a slope similar to the one in the classical Fermi reacceleration. Assuming $M \sim 1.6$, consistent with our findings in $\S 3.1$, a power-law tail of radio emission with $\alpha \sim 2.3$ can be generated at energies $\gamma>1000$ (Figure 8). This scenario avoids the previously mentioned energy argument, because the resulting steep spectrum of electrons does not extend down to the thermal energies.

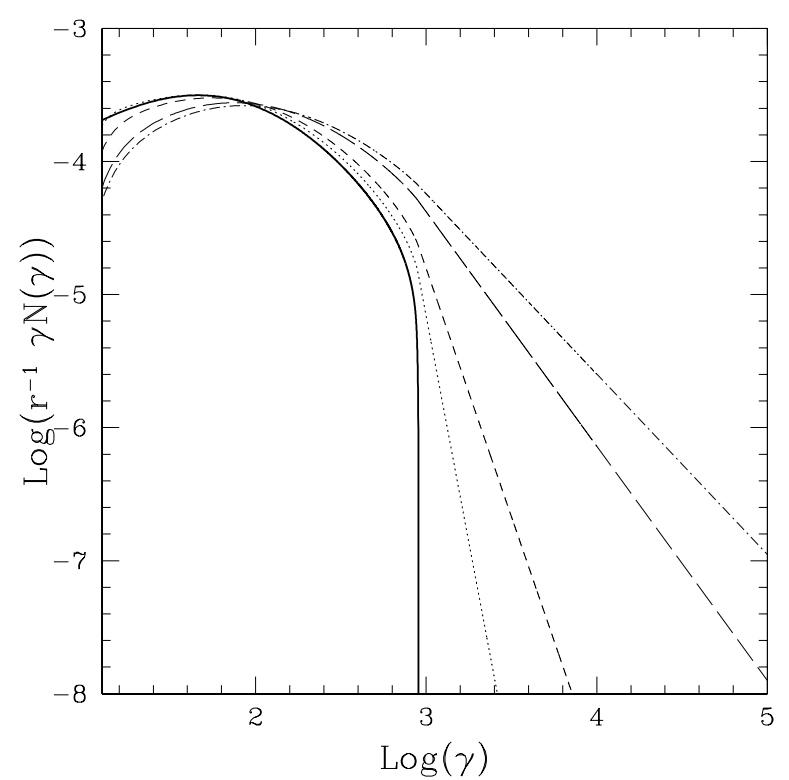

FIG. 8.- Spectrum of reaccelerated electrons according to Eq. 1]We also correct for the shock compression factor in the downstream region, $r$. The thick solid line is the initial spectrum of fossil electrons (assuming an age of the population of a few Gyrs and typical cluster physical parameters, e.g. Sarazin 2002). Different models show the spectrum for Mach numbers 1.3 (dotted line), 1.57 (dashed line), 2.5 (long-dashed line), and 3.5 (dot-dashed line). A minimum energy $\gamma_{\min }=10$ is adopted in the calculations.

3. The connection between the edge and the shock might be indirect. For instance, the passage of the shock may have driven small-scale turbulence in the ICM, which may be long-lived and also produce the radio halo on the larger scale. In this case, the radio edge would mark the region where turbulent acceleration is just beginning to occur. This should happen after $\sim 1$ eddy turnover-time of the turbulence, $\approx L / V_{l}$ (where $L$ and $V_{l}$ are the maximum scale and velocity of the turbulent eddies), since the turbulent modes at smaller scales are 
the most important in the particle acceleration process (e.g., Brunetti \& Lazarian 2007 and references therein). This may also explain the very steep spectrum of the radio emission in the shock region. A possible indirect connection between shocks and the cluster-wide diffuse radio emission is suggested by the present radio observation, as well as those of several other clusters, where bridges of emission connecting radio relics and halos in several clusters are seen (see, for instance, the discussion in Brunetti et al. 2008). A theoretical exploration of this scenario deserves a future effort.

\section{CONCLUSIONS}

We have presented a combined $\mathrm{X}$-ray and radio study of the nearby merging galaxy cluster Abell 754 .

The new Chandra observation confirms the presence of a merger shock front east of the cluster core by providing the Mach number from the density jump across the shock $\mathrm{M}=1.57_{-0.12}^{+0.16}$, and a direct measurement of a gas temperature jump of the right sign across the arc-like brightness edge, $T_{2} / T_{1}=2.2_{-0.7}^{+1.1}$.

The new GMRT radio image of the cluster at $330 \mathrm{MHz}$ reveals that the centrally located diffuse emission is very extended and complex, and is mainly elongated in the eastwest direction. Most interestingly, the eastern edge of this source coincides with the shock. We studied the spectral properties of the diffuse radio emission, using the GMRT data, archival $V L A$ data at $1.4 \mathrm{GHz}$, and previous $V L A$ results at $74 \mathrm{MHz}$. We find that the region next to the radio edge (the relic region in $\mathrm{K} 01$ ) has a very steep integrated spectrum, with $\alpha_{0.3 \mathrm{GHz}}^{1.4 \mathrm{GHz}}=2.02 \pm 0.04$ and $\alpha_{0.07 \mathrm{GHz}}^{0.3 \mathrm{GH}}=1.77 \pm 0.10$. This is steeper than the average spectrum of the whole diffuse emission, $\alpha_{0.3 \mathrm{GHz}}^{1.4 \mathrm{GH}}=1.57 \pm 0.05$, which is in itself steeper than the typical spectrum of radio halos.
The spatial coincidence of the steep spectrum radio edge and the merger shock front suggests a physical connection. The low Mach number of the shock and the very steep synchrotron spectrum of the edge allow us to rule out the scenario of direct shock acceleration of thermal particles for the origin of the edge, because it would require an implausible high acceleration efficiency (of order 1).

Possible alternative scenarios are reacceleration and/or adiabatic compression of fossil relativistic plasma in the ICM. Of these, shock reacceleration is more plausible for this weak shock. It should produce a spectrum of electrons that is in agreement with the observed steep radio spectrum of the relic region.

Finally, an indirect connection between the shock and the radio edge is also possible. The shock passage may drive smallscale turbulence that may reaccelerate electrons over the cluster volume.

Upcoming GMRT deep low-frequency observations will allow us to perform a more detailed study of the diffuse emission and help to determine the most plausible scenario for its origin.

GM thanks the hospitality of the Harvard-Smithsonian Center for Astrophysics where most of this work was done. We thank N. Kassim and T. Clarke for providing the VLA image at $74 \mathrm{MHz}$ and for useful comments. We thank Alexey Vikhlinin for stimulating discussions. We thank the staff of the GMRT for their help during the observations. GMRT is run by the National Centre for Radio Astrophysics of the Tata Institute of Fundamental Research. This research is partially funded by INAF under grant PRIN-INAF 2008, and by ASIINAF under grant E/088/06/0. Support was also provided by Chandra grant GO8-9128X and NASA contract NAS8-39073.

\section{REFERENCES}

Bacchi, M., Feretti, L., Giovannini, G., \& Govoni, F. 2003, A\&A, 400, 465 (B03)

Blandford, R., \& Eichler, D. 1987, Phys. Rep., 154, 1

Brentjens, M. A. 2008, A\&A, 489, 69

Brunetti, G., \& Lazarian, A. 2007, MNRAS, 378, 245

Brunetti, G., Giacintucci, S., Cassano, R., Lane, W., Dallacasa, D., Venturi, T., Kassim, N. E., Setti, G., Cotton, W. D., Markevitch, M. 2008, Nature, 455,944

Cassano, R. 2009, Astronomical Society of the Pacific Conference Series, 357,1313

Clarke, T. E., Ensslin, T. A. 2006, AJ, 131, 2900

Ensslin, T. A., Biermann, P. L., Klein, U., \& Kohle, S. 1998, A\&A, 332, 395

Enßlin, T. A., \& Gopal-Krishna 2001, A\&A, 366, 26

Fabricant, D., Beers, T. C., Geller, M. J., Gorenstein, P., Huchra, J. P., \& Kurtz, M. J. 1986, ApJ, 308, 530

Ferrari, C., Govoni, F., Schindler, S., Bykov, A. M., \& Rephaeli, Y. 2008, Space Science Reviews, 134, 93

Fusco-Femiano, R., Orlandini, M., De Grandi, S., Molendi, S., Feretti, L., Giovannini, G., Bacchi, M., \& Govoni, F. 2003, A\&A, 398, 441

Giacintucci, S., Venturi, T., Macario, G., et al. 2008, A\&A, 486, 347

Greisen, Eric W., Spekkens, Kristine, van Moorsel, Gustaaf A. 2009, AJ, 137,4718

Henriksen, M. J., \& Markevitch, M. L. 1996, ApJ, 466, L79

Henry, J. P., \& Briel, U. G. 1995, ApJ, 443, L9

Henry, J. P., Finoguenov, A., \& Briel, U. G. 2004, ApJ, 615, 181

Hoeft, M., Brüggen, M., \& Yepes, G. 2004, MNRAS, 347, 389

Kale, R., Dwarakanath, K. S. 2009, ApJ, 407, 237

Kalberla, P. M. W.; Burton, W. B.; Hartmann, Dap; Arnal, E. M.; Bajaja, E.; Morras, R.; PŽppel, W. G. L., A\&A 2005, 440, 775 (LAB Map)
Kassim, N. E., Clarke, T. E., Enßlin, T. A., Cohen, A. S., \& Neumann, D. M. 2001, ApJ, 559, 785 (K01)

Krivonos, R. A., Vikhlinin, A. A., Markevitch, M. L., \& Pavlinsky, M. N. 2003, Astronomy Letters, 29, 425

Macario, G., Venturi, T., Brunetti, G., Dallacasa, D., Giacintucci, S., Cassano, R., Bardelli, S., Athreya, R. 2010, 517, 43

Markevitch, Maxim, Sarazin, Craig L., Vikhlinin, Alexey 1999, ApJ, 521, 526

Markevitch, M., Ponman, T. J., Nulsen, P. E. J., Bautz, M. W., et al. 2000, ApJ, 541, 542

Markevitch, M., \& Vikhlinin, A. 2001, ApJ, 563, 95

Markevitch, M., Gonzalez, A. H., David, L., Vikhlinin, A., Murray, S., Forman, W., Jones, C., Tucker, W. 2002, ApJ, 567, 27

Markevitch, M., Bautz, M. W., Biller, B. et al. 2003b, ApJ, 583, 19

Markevitch, M., Mazzotta, P., Vikhlinin, A., et al. 2003, ApJ, 586, L19 (M03)

Markevitch, M., Govoni, F., Brunetti, G., \& Jerius, D. 2005, ApJ, 627, 733

Markevitch, M., Vikhlinin 2007, Physics Reports, 433, 1

Rephaeli, Yoel, Gruber, Duane 2002, ApJ, 579, 587

Roettiger, K., Stone, J. M., \& Mushotzky, R. F. 1998, ApJ, 493, 62

Russell, H. R., Sanders, J. S., Fabian, A. C., Baum, S. A., Donahue, M.,

Edge, A. C., McNamara, B. R., O’Dea, C. P. 1998, ApJ, 406, 1721

Sarazin, C. L. 2002, Astrophysics and Space Science Library, Vol. 272, 1

Struble, M. F., \& Rood, H. J. 1999, ApJS, 125, 35

Vikhlinin, A., Markevitch, M., \& Murray, S. S. 2001, ApJ, 551, 160

Vikhlinin, A., Markevitch, M., Murray, S. S., Jones, C.; Forman, W., Van Speybroeck, L. 2005, ApJ, 628, 655

Wik, Daniel R., Sarazin, Craig L., Finoguenov, Alexis, Matsushita, Kyoko,

Nakazawa, Kazuhiro, Clarke, Tracy E. 2009, ApJ, 696, 1700

Zabludoff, A. I., \& Zaritsky, D. 1995, ApJ, 447, L21 